\title{
Minimum-Length Polygons in Simple Cube-Curves
}

\author{
Reinhard Klette ${ }^{1}$ and Thomas Bülow ${ }^{2}$ \\ 1 CITR, University of Auckland, Tamaki Campus \\ Building 731, Auckland, New Zealand \\ r.klette@auckland.ac.nz \\ 2 GRASP Laboratory, University of Pennsylvania \\ 3401 Walnut Street, 330 C, Philadelphia, PA 19104-6228, USA \\ thomasbl@grasp.cis.upenn.edu
}

\begin{abstract}
Simple cube-curves in a 3D orthogonal grid are polyhedrally bounded sets which model digitized curves or arcs in three-dimensional euclidean space. The length of such a simple digital curve is defined to be the length of the minimum-length polygonal curve fully contained and complete in the tube of this digital curve. A critical edge is a grid edge contained in three consecutive cubes of a simple cube-curve. This paper shows that critical edges are the only possible locations of vertices of the minimum-length polygonal curve fully contained and complete in the tube of this digital curve.
\end{abstract}

\section{Introduction}

The estimation of the length of a simple digital curve in three-dimensional euclidean space may be based on the calculation of the shortest polygonal curve in a polyhedrally bounded compact set $[11,12]$. This paper presents an analysis of possible locations of vertices of such a polygonal curve. This analysis has been used in [2] for the design of an iterative algorithm approximating such curves with measured time complexity in $\mathcal{O}(n)$, where $n$ denotes the number of grid cubes of the given digital curve.

\subsection{Digital Curves in 3D Space}

Any grid point $(i, j, k) \in \mathcal{R}^{3}, i, j, k$ integers, is assumed to be the center point of a grid cube with faces parallel to the coordinate planes, with edges of length 1 , and vertices. Cells are either cubes, faces, edges or vertices. The intersection of two cells is either empty or a joint side of both cells. We consider a non-empty finite set $K$ of cells such that for any cell in $K$ it holds that any side of this cell is also in $K$. Such a set $K$ is a special finite euclidean complex [9]. Let $\operatorname{dim}(a)$ denote the dimension of a cell $a$, which is 0 for vertices, 1 for edges, 2 for faces and 3 for cubes. Then $[K, \subset, d i m]$ is also a cell complex $[5,7,9,13]$ with properties such as (i) $\subset$ is transitive on $K$, (ii) $\operatorname{dim}$ is monotone on $K$ with respect to $\subset$, and (iii) for any pair of cells $a, b \in K$ with $a \subset b$ and $\operatorname{dim}(a)+1<\operatorname{dim}(b)$ there

G. Borgefors, I. Nyström, and G. Sanniti di Baja (Eds.): DGCI 2000, LNCS 1953, pp. 467-478, 2000. (C) Springer-Verlag Berlin Heidelberg 2000 
exists a cell $c \in K$ with $a \subset c \subset b$. Cell $b$ bounds cell $a$ iff $a \subset b$, and $b$ is a proper side of $a$ in this case. Two cells $a$ and $b$ are incident iff cell $a$ bounds $b$, or cell $b$ bounds $a$.

We define digital curves $g$ in $3 \mathrm{D}$ space with respect to such a euclidean complex as special sequences $\left(z_{0}, z_{1}, \ldots, z_{m}\right)$ of cells where $z_{i}$ is incident with $z_{i+1}$, and $\left|\operatorname{dim}\left(z_{i}\right)-\operatorname{dim}\left(z_{i+1}\right)\right|=1$, for $i+1(\bmod m+1)$. There are (at least) three different options which may depend upon an application context, or upon a preference of either a grid-point model or a cellular model which are dual approaches [4]. Let $n \geq 1$.

(i) An edge-curve is a sequence $g=\left(v_{0}, e_{0}, v_{1}, e_{1}, \ldots, v_{n}, e_{n}\right)$ of vertices $v_{i}$ and edges $e_{i}$, for $0 \leq i \leq n$, such that vertices $v_{i}$ and $v_{i+1}$ are sides of edge $e_{i}$, for $0 \leq i \leq n$ and $v_{n+1}=v_{0}$. It is simple iff each edge of $g$ has exactly two bounding vertices in $g$. It follows that a vertex or edge is contained at most once in a simple edge curve. ${ }^{1}$

(ii) A face-curve is a sequence $g=\left(e_{0}, f_{0}, e_{1}, f_{1}, \ldots, e_{n}, f_{n}\right)$ of edges $e_{i}$ and faces $f_{i}$, for $0 \leq i \leq n$, such that edges $e_{i}$ and $e_{i+1}$ are sides of face $f_{i}$, for $0 \leq i \leq n$ and $e_{n+1}=e_{0}$. It is simple iff $n \geq 4$, and for any two faces $f_{i}, f_{k}$ in $g$ with $|\bar{i}-k| \geq 2(\bmod n+1)$ it holds that if $f_{i} \cap f_{k} \neq \emptyset$ then $|i-k|=2(\bmod n+1)$ and $f_{i} \cap f_{k}$ is a vertex.

(iii) A cube-curve is a sequence $g=\left(f_{0}, c_{0}, f_{1}, c_{1}, \ldots, f_{n}, c_{n}\right)$ of faces $f_{i}$ and cubes $c_{i}$, for $0 \leq i \leq n$, such that faces $f_{i}$ and $f_{i+1}$ are sides of cube $c_{i}$, for $0 \leq i \leq n$ and $\bar{f}_{n+1}=f_{0}$. It is simple iff $n \geq 4$, and for any two cubes $c_{i}, c_{k}$ in $g$ with $|i-k| \geq 2(\bmod n+1)$ it holds that if $c_{i} \cap c_{k} \neq \emptyset$ then either $|i-k|=2(\bmod n+1)$ and $c_{i} \cap c_{k}$ is an edge, or $|i-k|=3(\bmod n+1)$ and $c_{i} \cap c_{k}$ is a vertex. A tube $\mathbf{g}$ is the union of all cubes contained in a cubecurve $g$. It is a polyhedrally-bounded compact set in $\mathcal{R}^{3}$, and it is homeomorphic with a torus in case of a simple cube-curve. ${ }^{2}$

\subsection{MLP in 3D Space}

This paper deals exclusively with simple cube-curves. The cube-curve on the left of Fig. 1 is simple, and the cube-curve on the right is not. The latter example shows that the polyhedrally-bounded compact set $\mathbf{g}$ of a cube-curve $g$ is not necessarily homeomorphic with a torus if each cube of this cube-curve $g$ has exactly two bounding faces in $g$. A (Jordan) curve is complete in $\mathbf{g}$ iff it has a non-empty intersection with any cube contained in $g$.

Definition 1. A minimum-length polygon (MLP) of a simple cube-curve $g$ is a shortest polygonal simple curve $\mathcal{P}$ which is contained and complete in tube $\mathbf{g}$.

Following [11,12], the length of a simple cube-curve $g$ is defined to be the length $l(\mathcal{P})$ of an MLP of $g$.

\footnotetext{
1 This definition is consistent with, e.g., the definition of a 4-curve in [10] (see proposition 2.3.3) for 2D grids where our edges are 'hidden' in a neighborhood definition, or of a closed simple path in [14] (see page 7) for undirected graphs.

${ }^{2}$ Closed simple one-dimensional grid continua [11,12] are defined such that each cube of $g$ has exactly two bounding faces in $g$.
} 


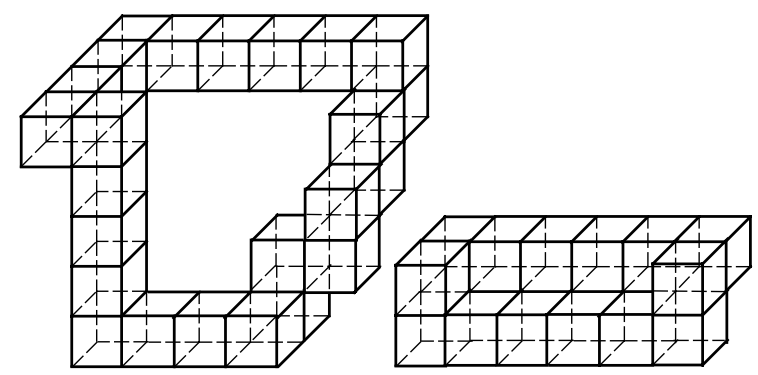

Fig. 1. Two cube-curves in 3D space

A simple cube-curve $g$ is flat iff the center points $(i, j, k)$ of all cubes contained in $g$ are in one plane parallel to one of the coordinate planes. A non-flat simple cube-curve in $\mathcal{R}^{3}$ specifies exactly one minimum-length polygonal simple curve (MLP, minimum-length polygon) which is contained and complete in its tube [11]. The MLP is not uniquely specified in flat simple cube-curves. Flat simple cube-curves may be treated as square-curves in the plane, and squarecurves in the plane are extensively studied, see, e.g. [6]. It seems there is no straightforward approach to extend known 2D algorithms to the $3 \mathrm{D}$ case. An important reason for that may be that $2 \mathrm{D}$ algorithms for (multigrid-convergent) perimeter estimation [6] may be such that all calculated vertices are grid points or vertices, but in the $3 \mathrm{D}$ case we are faced with a qualitatively new situation for the calculated vertices. The minimum-length polygon considered in this paper leads to vertices with real coordinates (not just multiples of integers as in the $2 \mathrm{D}$ case), i.e. the model of cell complexes is considered as being embedded into the euclidean space. However, independent upon the dimension global information has to be taken into account for length calculation of digital curves to ensure multigrid convergence.

\section{Simple Cube-Curves}

This section contains fundamental definitions and properties related to simple cube-curves.

\subsection{Non-contractible Curves in g}

Let $g$ be a simple cube-curve, and $\mathcal{P}=\left(p_{0}, p_{1}, \ldots, p_{m}\right)$ be a polygonal curve complete and contained in $\mathbf{g}$, with $p_{0}=p_{m}$.

Lemma 1. It holds $m \geq 3$, for any polygon $\mathcal{P}=\left(p_{0}, p_{1}, \ldots, p_{m}\right)$ complete and contained in a simple cube-curve. Two line segments alone cannot be complete in any simple cube-curve. 

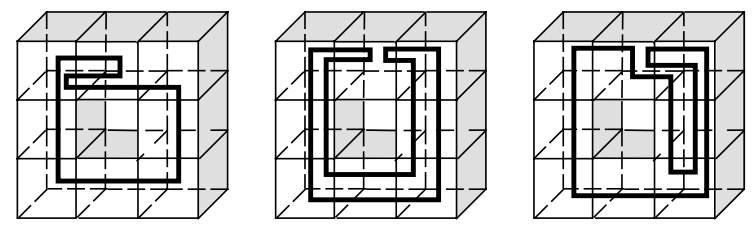

Fig. 2. Curves complete and contained in a tube

Proof. Cases $m=0$ and $m=1$ would be a point, and $m=2$ would be a straight line segment. Both cases are excluded because our simple cube-curves are homeomorphic to the torus. The case $m=3$ (a triangle) is possible, e.g. for the simple cube-curve shown in Fig. 2. However, in this minimum case of $m=3$ it holds that no side of the triangle may be completely contained within one of the cubes.

A curve contained in $\mathbf{g}$ is contractible (into a point) in $\mathbf{g}$ iff there are continuously repeated topological (i.e. continuous and bijective) transformations of this curve into a family of disjoint curves, all contained in $\mathbf{g}$, which converge towards a single point [8]. The curves on the left and on the right in Fig. 2 are non-contractible in $\mathbf{g}$, and the curve in the middle is contractible in $\mathbf{g}$ by continuous contractions into a single point.

A Jordan curve $\gamma$ passes through a face $f$ iff there are parameters $t_{1}, t_{2}, T$ such that $\left\{\gamma(t): t_{1} \leq t \leq t_{2}\right\} \subseteq f$, and $\gamma\left(t_{1}-\varepsilon\right) \notin f, \gamma\left(t_{2}+\varepsilon\right) \notin f$, for all $\varepsilon$ with $0<\varepsilon \leq T$. During a traversal along curve $\gamma$ we enter a cube $c$ at point $\gamma\left(t_{1}\right) \in c$ if $\gamma\left(t_{1}-\varepsilon\right) \notin c$, and we leave $c$ at point $\gamma\left(t_{2}\right) \in c$ if $\gamma\left(t_{2}+\varepsilon\right) \notin c$, for all $\varepsilon$ with $0<\varepsilon \leq T$. A traversal is defined by the starting vertex $p_{0}$ of the curve and the given orientation.

We consider polygonal curves $\mathcal{P}$. Let $\mathcal{C}_{\mathcal{P}}=\left(c_{0}, c_{1}, \ldots, c_{n}\right)$ be the sequence of cubes in the order how they are entered during curve traversal. If $\mathcal{P}$ is complete and contained in a tube $\mathrm{g}$ then it follows that $\mathcal{C}_{\mathcal{P}}$ contains all cubes of $g$, and there are no further cubes in $\mathcal{C}_{\mathcal{P}}$.

Lemma 2. For an $M L P \mathcal{P}$ of a simple cube-curve $g$ it holds that $\mathcal{C}_{\mathcal{P}}$ contains each cube of $g$ just once.

Proof. Assume that $\mathcal{P}$ enters the same cube $c$ of $g$ twice, say at point $q_{1}$ first and at point $q_{2}$ again. Both points may be on one face of $c$, see Fig. 2 on the left and on the right, or on different faces of $c$, see Fig. 2 middle.

First consider the case that both entry points $q_{1}$ and $q_{2}$ of $c$ are on one face $f$ of cubes $c$ and $c^{\prime}$. Assume the number of passes of $\mathcal{P}$ through $f$ is odd. We insert points $q_{1}$ and $q_{2}$ into $\mathcal{P}$ as new vertices which split the resulting polygonal curve into two polygonal chains, $\mathcal{P}_{1}=\left(q_{2}, \ldots, q_{1}\right)$ and $\mathcal{P}_{2}=\left(q_{1}, \ldots, q_{2}\right)$ such that the union of both is $\mathcal{P}$. The length of $\mathcal{P}_{i}$ exceeds the length of the straight line segment $q_{1} q_{2}$, for $i=1,2$. W.l.o.G. let $\mathcal{P}_{1}$ be the chain which does not pass through $f$. It follows that $\mathcal{P}_{1}$ is complete in $\mathrm{g}$. Because the cube $c$ is convex it 
also contains the straight line segment $q_{1} q_{2}$. We replace the polygonal sequence $\mathcal{P}_{2}$ by $q_{1} q_{2}$, i.e. we replace $\mathcal{P}$ by $\mathcal{Q}=\left(q_{1}, q_{2}, \ldots, q_{1}\right)$. Curve $\mathcal{Q}$ is still complete and contained in $\mathrm{g}$, but shorter than $\mathcal{P}$ which contradicts our assumption that $\mathcal{P}$ is an MLP of $g$.

Now assume that the number of passes of $\mathcal{P}$ through $f$ is even, it enters $c$ at $q_{1}$, then it passes $f$ and enters $c^{\prime}$ at $r_{1}$, then it passes $f$ again and enters $c$ at $q_{2}$, then it passes $f$ again and enters $c^{\prime}$ at $r_{2}$. There may be a further even number of passes of $\mathcal{P}$ through $f$ before the curve returns to $q_{1}$. We insert points $q_{1}, r_{1}, q_{2}, r_{2}$ into $\mathcal{P}$ as new vertices which split the resulting polygonal curve into four polygonal chains, $\mathcal{P}_{1}=\left(q_{1}, \ldots, r_{1}\right), \mathcal{P}_{2}=\left(r_{1}, \ldots, q_{2}\right)$, $\mathcal{P}_{3}=\left(q_{2}, \ldots, r_{2}\right)$ and $\mathcal{P}_{2}=\left(r_{2}, \ldots, q_{1}\right)$ such that the union of all four is $\mathcal{P}$. It follows that

$$
\mathcal{C}_{\mathcal{P}_{1}} \subseteq \mathcal{C}_{\mathcal{P}_{3}} \vee \mathcal{C}_{\mathcal{P}_{3}} \subseteq \mathcal{C}_{\mathcal{P}_{1}}
$$

and an analog conclusion for $\mathcal{P}_{2}$ and $\mathcal{P}_{4}$. W.l.o.g. let $\mathcal{C}_{\mathcal{P}_{1}} \subseteq \mathcal{C}_{\mathcal{P}_{3}}$. Then we replace in $\mathcal{P}$ the polygonal chain $\mathcal{P}_{1}$ by the straight line segment $q_{1} r_{1}$ which is in $f$. The length of $\mathcal{P}_{1}$ exceeds the length of the straight line segment $q_{1} r_{1}$. Thus the resulting polygonal curve is still complete and contained in $\mathbf{g}$, but shorter than $\mathcal{P}$ which also contradicts our assumption that $\mathcal{P}$ is an MLP of $g$.

We consider the second case that both points $q_{1}$ and $q_{2}$ are on different faces of cube $c$, say $q_{1}$ on face $f_{1}$ and $q_{2}$ on face $f_{2}$. Because $q_{2}$ is a re-entry point to cube $c$ there must be a point $q_{e x}$ in $f_{2}$ where we leave $c$ before entering $c$ again at $q_{2}$. If there is another re-entry point on face $f_{2}$ then we are back to case one. It follows that $\mathcal{P}$ leaves $c$ once and enters $c$ once. Assume that $f_{2}$ is also a face of cube $c^{\prime} \neq c$ of $g$. If $\mathcal{P}$ would not intersect the second face of $c^{\prime}$ contained in $g$ then we may replace the polygonal subsequence $\left(q_{e x}, \ldots, q_{2}\right)$ (which is contained in $c^{\prime}$ but not in $f_{2}$ ) by the shorter straight line segment $q_{e x} q_{2}$ which is contained in $f_{2}$ and thus in $c^{\prime}$, i.e. the resulting polygonal curve would be shorter and still contained and complete in $g$. It follows that the curve $\mathcal{P}$ has to leave cube $c^{\prime}$ through its second face contained in $g$. Tracing $g$ around means that we arrive at the cube $c^{\prime \prime} \neq c$ which is also incident with face $f_{1}$, and we leave $c^{\prime \prime}$ (and enter $c$ ) at a point which may be equal to $q_{1}$, and we enter $c^{\prime \prime}$ again through $f_{1}$. Thus $\mathcal{P}$ contains two polygonal subsequences which are both contained and complete in $g$. This contradicts the shortest-length constraint.

\subsection{Iterative Modifications}

Now we consider a special transformation of polygonal curves. Let $\mathcal{P}=$ $\left(p_{0}, p_{1}, \ldots, p_{m}\right)$ be a polygonal curve contained in a tube $\mathbf{g}$. A polygonal curve $\mathcal{Q}$ is a $\mathbf{g}$-transform of $\mathcal{P}$ iff $\mathcal{Q}$ may be obtained from $\mathcal{P}$ by a finite number of steps, where each step is a replacement of a triple $a, b, c$ of vertices by a polygonal sequence $a, b_{1}, \ldots, b_{k}, c$ such that the polygonal sequence $a, b_{1}, \ldots, b_{k}, c$ is contained in the same set of cubes of $g$ as the polygonal sequence $a, b, c$. The case $k=0$ characterizes the deletion of vertex $b$, the case $k=1$ characterizes a move of vertex $b$ within $\mathbf{g}$, and cases $k \geq 2$ specify a replacement of two straight line segments by a sequence of $k+1$ straight line segments, all contained in $\mathbf{g}$. 


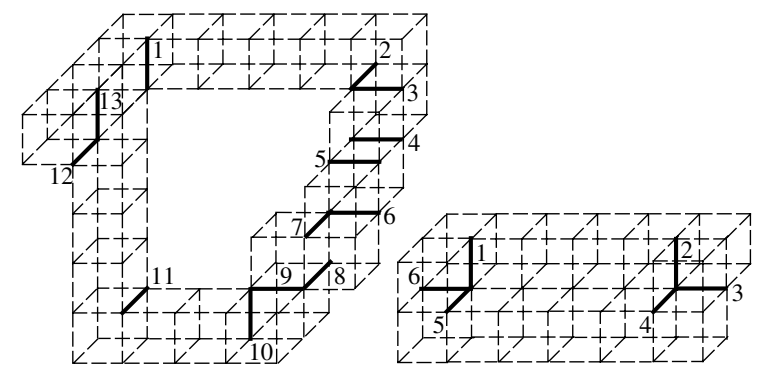

Fig. 3. Critical edges of two cube-curves

Lemma 3. Let $\mathcal{P}$ be a polygonal curve complete and contained in the tube $\mathbf{g}$ of a simple cube-curve $g$ such that $\mathcal{C}_{\mathcal{P}}$ is without repetitions of cells. Then it holds that any $\mathbf{g}$-transform of $\mathcal{P}$ is also complete and contained in $\mathbf{g}$.

Proof. By definition of the $\mathrm{g}$-transform it follows that this curve is also contained in $\mathrm{g}$. Because $\mathcal{C}_{\mathcal{P}}$ is without repetitions of cells it holds that $\mathcal{P}$ traces $\mathrm{g}$ cell by cell, starting with one vertex in one cell and returning to the same vertex. From Lemma 1 we know that $\mathcal{P}$ has at least three vertices, i.e. at least three line segments, and that for the minimum case of $m=3$ it holds that two line segments cannot be complete in $g$, i.e. there is at least one cube not intersected by these two line segments. Thus a replacement of two line segments (within the same set of cells of $g$ ) cannot transform $\mathcal{P}$ into a curve contractible in $\mathrm{g}$, i.e. the curve remains complete in $\mathbf{g}$.

\section{Critical Edges}

An edge contained in a tube $\mathbf{g}$ is critical iff this edge is the intersection of three cubes contained in the cube-curve $g$. Figure 3 illustrates all critical edges of the cube-curves shown in Fig. 1. Note that simple cube-curves may only have edges contained in three cubes at most. For example, the cube-curve consisting of four cubes only (note: there is one edge contained in four cubes in this case) was excluded by the constraint $n \geq 4$.

Theorem 1. Let $g$ be a simple cube-curve. Critical edges are the only possible locations of vertices of a shortest polygonal simple curve contained and complete in tube $\mathbf{g}$.

Proof. We consider arbitrary (flat or non-flat) simple cube-curves $g$, i.e. the MLP may not be uniquely defined.

Let $\mathcal{P}=\left(p_{0}, p_{1}, \ldots, p_{m}\right)$ be a shortest polygonal simple curve contained and complete in tube $\mathbf{g}$, with $p_{0}=p_{m}$ and $m \geq 3$. We consider w.l.o.g. the polygonal subsequence $\left(p_{0}, p_{1}, p_{2}\right)$ of such a shortest polygonal simple curve contained and complete in tube $\mathbf{g}$. We will show that $p_{1}$ is on a critical edge. According to 


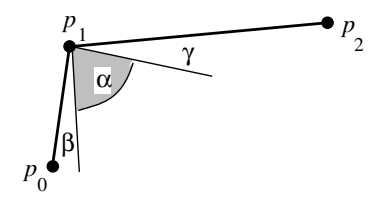

Fig. 4. Sketch of point $p_{1}$

Lemma 2 we know that $\mathcal{C}_{\mathcal{P}}$ is without repetitions, i.e. we may apply Lemma 3 for this curve $\mathcal{P}$ and tube $\mathbf{g}$.

We can exclude the case that $p_{1}$ is collinear with $p_{0}$ and $p_{2}$, because $p_{1}$ would be no vertex of a polygon in such a case. Three non-collinear points $p_{0}, p_{1}$, and $p_{2}$ define a triangular region $\triangle\left(p_{0}, p_{1}, p_{2}\right)$ in a plane $\mathcal{E}$ in $\mathcal{R}^{3}$. The following considerations are all for geometric configurations within this plane $\mathcal{E}$. In this proof, a boundary point is a point on the boundary $\partial \mathbf{g}$.

At first we ask whether $p_{1}$ may be moved into a new point $p_{n e w}$ within the triangle $\triangle\left(p_{0}, p_{1}, p_{2}\right)$ towards line segment $p_{0} p_{2}$ such that a resulting polygonal subsequence $\left(p_{0}, \ldots, p_{\text {new }}, \ldots, p_{2}\right)$ remains to be contained in $\mathbf{g}$. This describes a g-transform of $\mathcal{P}$, and the resulting curve would be complete and contained in g. It can be of shorter length if the intersection of an $\varepsilon$-neighborhood of $p_{1}$ with $\triangle\left(p_{0}, p_{1}, p_{2}\right)$ is in $\mathbf{g}$, for $\varepsilon>0$. It follows that such a move of $p_{1}$ is impossible, i.e. it follows that for any $\varepsilon>0$ there is at least one boundary point $q$ in an $\varepsilon$-neighborhood of $p_{1}$ and on one of the line segments $p_{0} p_{1}$ or $p_{1} p_{2}$, avoiding such a move of $p_{0}$ into the triangle $\triangle\left(p_{0}, p_{1}, p_{2}\right)$. It follows that $p_{1}$ itself is a boundary point.

The situation of an $\varepsilon_{0}$-neighborhood at point $p_{1}$ is illustrated in Fig. 4 . Angle $\alpha$ represents the region not in $\mathbf{g}$. Angles $\beta$ and $\gamma$ are just inserted to mention that they may be zero, and their actual value is not important in the sequel. It holds $\alpha<\pi$ because it is bounded by an inner angle of the triangle $\triangle\left(p_{0}, p_{1}, p_{2}\right)$.

A boundary point may be a point within a face, or on an edge. Assume first that boundary point $p_{1}$ is within a face $f$. Plane $\mathcal{E}$ and face $f$ either intersect in a straight line segment, or face $f$ is contained in $\mathcal{E}$. The straight line situation would contradict that $\alpha<\pi$ in the $\varepsilon_{0}$-neighborhood at point $p_{1}$, and $f \subset \mathcal{E}$ would allow to move $p_{1}$ into a new point $p_{\text {new }}$ within $\triangle\left(p_{0}, p_{1}, p_{2}\right)$ towards line segment $p_{0} p_{2}$ which contradicts our MLP assumption.

There are three different possibilities for an edge contained in $\mathrm{g}$ : we call it an uncritical edge if it is only in one cube contained in $g$, it is an ineffective edge if it is in exactly two cubes contained in $g$, and it is a critical edge (as defined above) in case of three cubes. Point $p_{1}$ cannot be on an ineffective edge such that it is also not on a critical or uncritical edge, because this corresponds to the situation being within a face as discussed before. Point $p_{1}$ also cannot be on an uncritical edge such that it is also not on a critical edge. Figure 5 illustrates an intersection point $q$ with an uncritical edge in plane $\mathcal{E}$ assuming that this edge is not coplanar with $\mathcal{E}$. The resulting angle $\alpha>\pi$ (region not in $\mathbf{g}$ in an 
$\varepsilon$-neighborhood of $q$ ) does not allow that $p_{1}$ is such a point. If the uncritical edge is in $\mathcal{E}$ then angle $\alpha$ would be equal to $\pi$, what is excluded at $p_{1}$ as well. So there is only one option left. Point $p_{1}$ has to be on a critical edge (in fact, the angle $\alpha$ is less than $\pi$ for such an edge).

Note that this theorem also covers flat simple cube-curves with a straightforward corollary about the only possible locations of MLP vertices within a simple square-curve in the plane (see Fig. 6): such vertices may be convex vertices of the inner frontier or concave vertices of the outer frontier only because these are the only vertices incident with three squares of a simple square-curve.

\section{Application of the Theorem}

Our algorithm [2] is based on the following physical model: Assume a rubber band is laid through the tube g. Letting it move freely it will contract to the MLP which is contained and complete in $\mathbf{g}$ (assumed the band is slippery enough to slide across the critical edges of the tube). The algorithm consists of two subprocesses: at first an initialization process defining a simple polygonal curve $\mathcal{P}_{0}$ contained and complete in the given tube $\mathrm{g}$ and such that $\mathcal{C}_{\mathcal{P}_{0}}$ contains each cube of $g$ just once (see Lemma 2), and second an iterative process (a $\mathbf{g}$ transform, see Lemma 3) where each completed run transforms $\mathcal{P}_{t}$ into $\mathcal{P}_{t+1}$ with $l\left(\mathcal{P}_{t}\right) \geq l\left(\mathcal{P}_{t+1}\right)$, for $t \geq 0$. Thus the obtained polygonal curve is also complete and contained in $g$. This algorithm uses the fact that critical edges are the only possible locations of vertices of the desired polygonal curve. This allowed us to achieve linear running time.

\subsection{Initialization on Critical Edges}

We sketch the initialization procedure to illustrate the importance of the proved theorem. The initial polygonal curve will only connect vertices which are end points of consecutive critical edges. For curve initialization, we scan the given curve until the first pair $\left(e_{0}, e_{1}\right)$ of consecutive critical edges is found which are not parallel or, if parallel, not in the same grid layer (see Fig. 1 (right) for a non-simple cube-curve showing that searching for a pair of non-coplanar edges would be insufficient in this case). For such a pair $\left(e_{0}, e_{1}\right)$ we start with vertices $\left(p_{0}, p_{1}\right), p_{0}$ bounds $e_{0}$ and $p_{1}$ bounds $e_{1}$, specifying a line segment $p_{0} p_{1}$ of

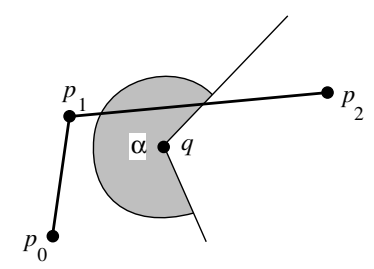

Fig. 5. Intersection with an uncritical edge 


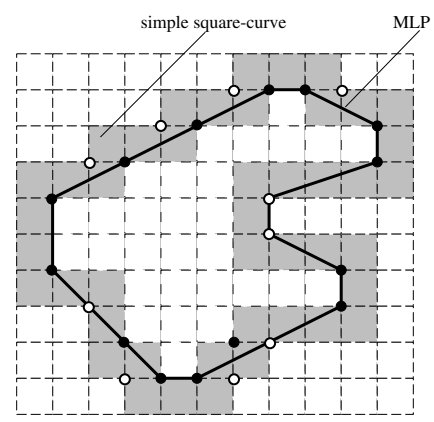

- convex vertices of inner frontier

○ concave vertices of outer frontier

Fig. 6. Convex vertices of inner and concave vertices of outer frontier of the tube of a simple square curve in the plane

minimum length (note that such a pair $\left(p_{0}, p_{1}\right)$ is not always uniquely defined). This is the first line segment of the desired initial polygonal curve $\mathcal{P}_{0}$.

Now assume that $p_{i-1} p_{i}$ is the last line segment on this curve $\mathcal{P}_{0}$ specified so far, and $p_{i}$ is a vertex which bounds $e_{i}$. Then there is a uniquely specified vertex $p_{i+1}$ on the following critical edge $e_{i+1}$ such that $p_{i} p_{i+1}$ is of minimum length. Length zero is possible with $p_{i+1}=p_{i}$; in this case we skip $p_{i+1}$, i.e. we do not increase the value of $i$. Note that this line segment $p_{i} p_{i+1}$ will always be included in the given tube because the centers of all cubes between two consecutive critical edges are collinear. The process stops by connecting $p_{n}$ on edge $e_{n}$ with $p_{0}$ (note that it is possible that a minimum-distance criterion for this final step may actually prefer a line between $p_{n}$ and the second vertex bounding $e_{0}$, i.e. not $p_{0}$ ). See Table 1 for a list of calculated vertices for the cubecurve on the left in Figs. 1 and 3. The first row lists all the critical edges shown in Fig. 3. The second row contains the vertices of the initial polygon shown in Fig. 7 (initialization $=$ first run of the algorithm). For example, vertex $b$ is on edge 2 and also on edge 3 , so there is merely one column for $(2 / 3)$ for these edges.

This initialization process calculates a polygonal curve $\mathcal{P}_{0}$ which is always contained and complete in the given tube. Note that traversals following opposite orientations or starting at different critical edges may lead to different initial polygons. For example, a 'counterclockwise' scan of the cube-curve shown in Fig. 1 (left), starting at edge 1, selects edges 11 and 10 to be the first pair of

Table 1. Calculated points on edges (' $\mathrm{D}$ ' in stands for 'deletion', i.e. there is no polygon vertex on this edge anymore)

\begin{tabular}{|c|c|c|c|c|c|c|c|c|c|c|}
\hline critical edge & 1 & $2 / 3$ & 4 & 5 & $6 / 7$ & 8 & 9 & 10 & 11 & $12 / 13$ \\
\hline 1 st run (initialization) & $\mathrm{a}$ & $\mathrm{b}$ & $\mathrm{c}$ & $\mathrm{d}$ & $\mathrm{e}$ & $\mathrm{f}$ & $\mathrm{g}$ & $\mathrm{h}$ & $\mathrm{i}$ & $\mathrm{j}$ \\
\hline 2nd run & $\mathrm{a}$ & $\mathrm{b}$ & $\mathrm{D}$ & $\mathrm{D}$ & $\mathrm{e}$ & $\mathrm{D}$ & $\mathrm{D}$ & $\mathrm{h}$ & $\mathrm{i}$ & $\mathrm{j}$ \\
\hline
\end{tabular}




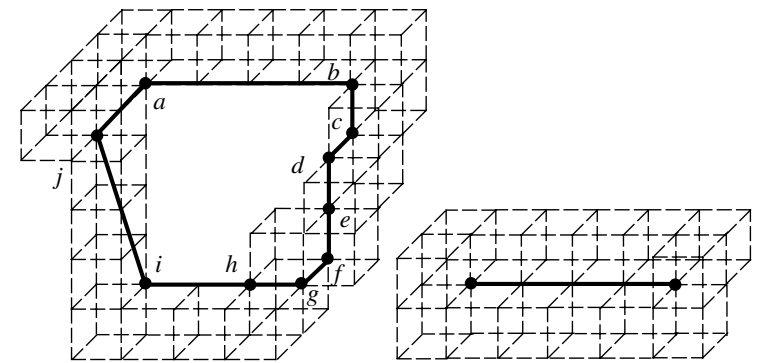

Fig. 7. Curve initializations ('clockwise')

consecutive critical edges, and the generated 'counterclockwise' polygon would differ from the one shown in Fig. 7. Figure 8 shows a curve where the defined initialization does not return to the starting vertex.

Initialization results are shown in Figs. 8 and 9. Note that in case of flat cube-curves the process will fail to determine the specified first pair of critical edges, and in this case a 2D algorithm may be used to calculate the MLP of a corresponding square-curve.

\subsection{Non-grid-point Vertices of MLP's}

This initialization procedure is followed by an iterative procedure [2] where we move pointers addressing three consecutive vertices of the (so far) calculated polygonal curve around the curve, until a completed run $t+1$ does only lead to an improvement which is below an a-priori threshold $\tau$ i.e. $l\left(\mathcal{P}_{t}\right)-\tau<l\left(\mathcal{P}_{t+1}\right)$.

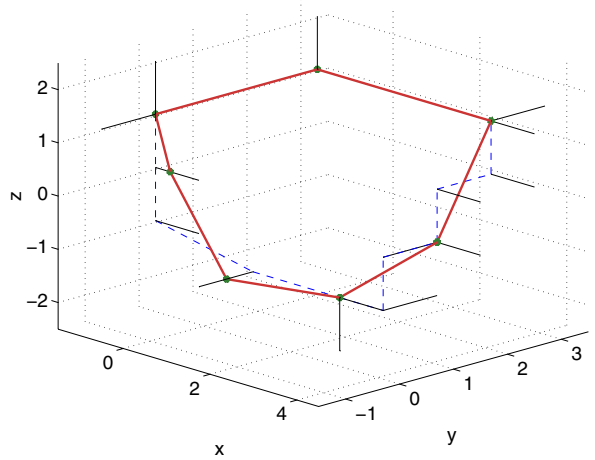

Fig. 8. Initial polygon (dashed) and MLP. Initialization starts below on the left, and the final step of the initialization process would prefer the second vertex of the first edge if a shortest-distance criterion would be used only 


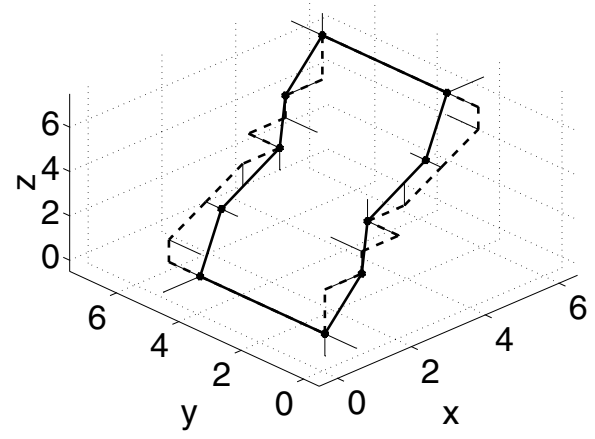

Fig. 9. Initial polygon (dashed) and MLP. Critical edges are shown as short line segments. The rest of the tube is not shown

Figures 8 and 9 show the initial polygon $\mathcal{P}_{1}$ dashed, and the solid line represents the final polygon. The short line segments are the critical edges of the given tube. For details of the algorithm see [2]. Note that MLP vertices may also be in "non-vertex" positions dividing a critical edge into two segments of non-zero (rational) length.

\section{Conclusions}

Length-estimators for digital curves in the plane are a well-studied subject. See, for example, [6], for references in this field. There are methods for length estimation in the digital plane which are provable convergent to the true length value for specific convex planar sets, assuming finer and finer grids in the plane. Such a correct convergence behavior is also supported by experimental evidence [6]. Methods showing multigrid convergence in the digital plane are, for example, the 'classical' digital straight line approximation technique (DSS method), the gridcontinua based minimum-length polygon method (GC-MLP method), see [11], and a minimum-length polygon approximation method based on so-called approximation sausages (AS-MLP method), see [1] for this AS-MLP method.

Length-estimators for digital curves in the 3D space may be designed by following $2 \mathrm{D}$ design principles. The discussed method in this paper expands the GC-MLP method, see [12]. Due to this fact it follows that the discussed method satisfies multigrid convergence for the special case of flat curves as the 2D method does for planar curves. This might be sufficient theoretical evidence for the convergence behavior of the discussed curve length estimation method.

The paper has specified an important geometric property of such minimumlength polygons in 3D space which has been used in [2] for designing an efficient algorithm for calculating such a polygon. The given theorem has been used in [2] but without proof and accompanying definitions. In this sense the paper 
has provided fundamentals for the algorithm discussed in [2]. The given theorem might be also of interest in the context of $3 \mathrm{D}$ curves in general.

\section{References}

1. T. Asano, Y. Kawamura, R. Klette, K. Obokata: A new approximation scheme for digital objects and curve length estimations. CITR-TR 65, CITR Tamaki, The University of Auckland, August 2000. 477

2. Th. Bülow and R. Klette. Rubber band algorithm for estimating the length of digitized space-curves. Accepted paper for ICPR'2000, Barcelona, September 2000. 467, 474, 476, 477, 478

3. R. Busemann and W. Feller. Krümmungseigenschaften konvexer Flächen. Acta Mathematica, 66:27-45, 1935.

4. R. Klette. M-dimensional cellular spaces. Tech. Report TR-1256, Univ. of Maryland, Computer Science Dep., March 1983. 468

5. R. Klette. Cellular complexes through time. SPIE Conference Proceedings Vision Geometry IX, 4117:to appear, 2000. 467

6. R. Klette, V. Kovalevsky, and B. Yip. On the length estimation of digital curves. SPIE Conference Proceedings Vision Geometry VIII, 3811:117-129, 1999. 469, 477

7. V. Kovalevsky. Finite topology and image analysis. Advances in Electronics and Electron. Physics, 84:197-259, 1992. 467

8. J. B. Listing: Der Census räumlicher Complexe oder Verallgemeinerungen des Euler'schen Satzes von den Polyëdern. Abhandlungen der Mathematischen Classe der Königlichen Gesellschaft der Wissenschaften zu Göttingen 10 (1861 and 1862) 97-182. 470

9. W. Rinow. Topologie. Deutscher Verlag der Wissenschaften, Berlin, 1975. 467

10. A. Rosenfeld. Picture Languages. Academic Press, New York, 1979. 468

11. F. Sloboda and Ľ. Bačík. On one-dimensional grid continua in $R^{3}$. Report of the Institute of Control Theory and Robotics, Bratislava, 1996. 467, 468, 469, 477

12. F. Sloboda, B. Zaťko, and R. Klette. On the topology of grid continua. SPIE Conference Proceedings Vision Geometry VII, 3454:52-63, 1998. 467, 468, 477

13. A. W. Tucker. An abstract approach to manifolds. Annals of Math., 34:191-243, 1933. 467

14. K. Voss. Discrete Images, Objects, and Functions in $Z^{n}$. Springer, Berlin, 1993. 468 